\title{
Pulsed Vincristine Therapy in Steroid-Resistant Nephrotic Syndrome
}

\section{Shenal Thalgahagoda, ${ }^{1}$ Shamali Abeyagunawardena, ${ }^{2}$ Heshan Jayaweera, ${ }^{1}$ Umeshi Ishanthika Karunadasa, ${ }^{1}$ and Asiri Samantha Abeyagunawardena ${ }^{1}$}

\author{
${ }^{1}$ Department of Paediatrics, Faculty of Medicine, University of Peradeniya, Kandy, Sri Lanka
}

${ }^{2}$ Outpatient Department, Teaching Hospital Peradeniya, Peradeniya, Sri Lanka

Correspondence should be addressed to Asiri Samantha Abeyagunawardena; asiriabey26@gmail.com

Received 2 March 2017; Accepted 3 May 2017; Published 29 May 2017

Academic Editor: Jun Oh

Copyright (C) 2017 Shenal Thalgahagoda et al. This is an open access article distributed under the Creative Commons Attribution License, which permits unrestricted use, distribution, and reproduction in any medium, provided the original work is properly cited.

\begin{abstract}
Steroid-resistant nephrotic syndrome (SRNS) poses a therapeutic challenge for the paediatric nephrologist. As relentless progression to renal failure occurs with continued proteinuria, such patients will be treated with different cytotoxic medications with variable success rates and side-effects. We present here our findings on administering the anticancer drug vincristine for SRNS patients at a single centre in Sri Lanka. Methods. Between 2002 and 2007, fifty-four children presenting with steroid and cyclophosphamide resistance were treated with vincristine at $1.5 \mathrm{mg} / \mathrm{m}^{2}$ in weekly intravenous pulses for 8 weeks along with a tapering steroid regimen of 6 months. All patients were closely followed up for 5 years. Results. Of the 54 patients 39 were males and 15 were females (age range 3.5-11.6 years, median 6.1 years). At the end of the treatment course, 21 patients achieved complete remission while 7 had partial remission and no response was seen in 26 patients. Sustained remission at 6, 12, 24, and 60 months were 15 (27.78\%), 11 (20.37\%), 9 (16.67\%), and 7 (12.96\%), respectively. Most side-effects observed were reversible and no serious side-effects were noted during vincristine therapy. Conclusion. Although its therapeutic mechanisms in nephrotic syndrome are still not elucidated, vincristine appears to be a potent alternative that could be considered for treating SRNS.
\end{abstract}

\section{Introduction}

Nephrotic syndrome (NS) is the commonest paediatric glomerular disorder with an annual incidence of 2-7 per 100,000 [1]. While $80-90 \%$ children with NS achieve remission with initial corticosteroid therapy, the remaining $10-20 \%$ do not respond, thus being classified as steroid-resistant nephrotic syndrome (SRNS). A patient is considered to have steroid resistance if there is lack of remission despite treatment with prednisolone at a dose of $2 \mathrm{mg} / \mathrm{kg} /$ day $\left(60 \mathrm{mg} / \mathrm{m}^{2} /\right.$ day) for 4 weeks [2]. Due to the complications of unremitting proteinuria and progressive renal disease and the side-effects of treatment with immunosuppressive medication, the management of SRNS is difficult and challenging. Failure to induce remission carries a significant risk of progression to end-stage renal disease (ESRD) within 15 years in about $50 \%$ [3].
A renal biopsy is usually undertaken in all children with SRNS before starting specific treatment. Though the renal histology of most patients with steroid sensitive nephrotic syndrome (90\%) reveals minimal change nephropathy (MCN), the renal histology in SRNS is different, with up to $30-40 \%$ of patients showing focal segmental glomerulosclerosis (FSGS) [4]. The histologies in the remaining patients with SRNS include minimal change disease (30-40), mesangial proliferation, membranoproliferative glomerulonephritis, membranous nephropathy, and IgA nephropathy [2].

Children with SRNS have been treated with immunosuppressive agents such as cyclophosphamide (CYC), chlorambucil, and cyclosporine $\mathrm{A}(\mathrm{CYA})$ and more lately with mycophenolate mofetil. In those who do not respond or respond only partially, nonimmunosuppressive agents such as ACE inhibitors and angiotensin receptor blockers are employed to reduce the proteinuria. 
Cyclophosphamide, although widely used in the past to treat SRNS, is now thought to have little therapeutic efficacy in the treatment of this condition. Its efficacy seems to be more in those with minimal change disease and late steroid resistance and those with a partial response to steroids. It also possesses a sinister adverse effect profile including leucopenia, haemorrhagic cystitis, reversible alopecia, gonadal toxicity, and oncological risk $[5,6]$. At the time of the current study CYC was the primarily used agent for the treatment of SRNS.

CYA, a calcineurin inhibitor, has largely replaced CYC as the agent of choice for the treatment of SRNS, achieving significant complete remission rates [7]. It is effective in both minimal change disease and FSGS. This agent was however not freely available in Sri Lankan hospitals at the time of this study. Therefore other means of therapy had to be sought when patients presented with resistance to both corticosteroids and CYC. In this study we assess the efficacy of vincristine sulphate, a vinca alkaloid used in cancer therapy, in inducing and sustaining remission in SRNS.

\section{Patients and Methods}

This single-centre study was conducted at the Paediatric Nephrology Unit, Teaching Hospital Peradeniya, Sri Lanka. Children who failed to enter remission with prednisolone prescribed at a dose of $2 \mathrm{mg} / \mathrm{kg} /$ day $\left(60 \mathrm{mg} / \mathrm{m}^{2} /\right.$ day $)$ for 4 weeks were referred for further management. In all patients the same steroid dose was continued for additional 2 weeks during which a renal biopsy was performed. Patients who had a renal histology of either minimal change disease, idiopathic mesangial proliferation, or focal and segmental glomerulosclerosis were treated with oral cyclophosphamide (CYC) prescribed at a dose of $3 \mathrm{mg} / \mathrm{Kg} /$ day for 8 weeks along with $60 \mathrm{mg} / \mathrm{m}^{2}$ of alternate day steroids. The steroids were tapered over a period of 6 months. If remission was not achieved by 6 weeks of CYC therapy then CYC therapy was discontinued. These patients received vincristine at $1.5 \mathrm{mg} / \mathrm{m}^{2}$ in weekly intravenous pulses for 8 weeks along with a tapering course of steroids. The tapering steroid course consisted of $60 \mathrm{mg} / \mathrm{m}^{2}$ every other day for 2 weeks and then was tapered by $10 \mathrm{mg} / \mathrm{m}^{2}$ every 2 weeks over a period of 12 weeks. During therapy patients were reviewed on a weekly basis with full blood counts, urine protein excretion, serum protein and cholesterol levels, renal and liver function tests, and a full clinical examination focusing on the potential side-effects of vincristine. All possible side-effects were documented. Once they completed vincristine therapy, these patients were reviewed on a monthly basis.

We analysed the number of patients treated with vincristine from 2002 to 2007 who had complete, partial, or no remission along with the duration of sustained remission. We also analysed the adverse event profile during therapy. The collected data was entered in SPSS software version 16 and analysed using descriptive statistics and Mann-Whitney $U$ test.
TABLE 1: Baseline characteristics at the beginning of the study.

\begin{tabular}{lc}
\hline Characteristics & Value \\
\hline Number of patients & 54 \\
Median age (years) & 6.1 \\
Gender: & \\
$\quad$ Male & $39(72.2 \%)$ \\
$\quad$ Female & $15(27.8 \%)$ \\
\hline Biopsy histology: FSGS & $32(59.3 \%)$ \\
MCN and mesangial proliferation & $22(40.7 \%)$ \\
\hline
\end{tabular}

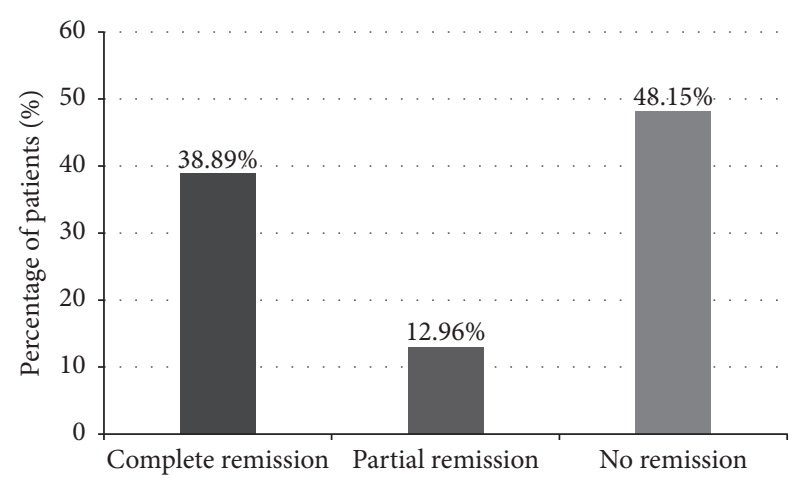

FIGURE 1: Patients achieving complete, partial, or no remission after vincristine therapy.

\section{Results}

The outcome of fifty-four children who received vincristine during this period was analysed. The ages ranged from 3.5 years to 11.6 years with a median of 6.1 years. Thirty-nine were males $(72.2 \%)$ and 15 were females $(27.8 \%)$. The baseline characteristics at the beginning of the study are shown in Table 1.

At the end of the course of vincristine, 21 patients out of the 54 achieved complete remission. Seven achieved partial remission and remission was not achieved in 26 patients (Figure 1).

Out of the patients who achieved complete remission at the end of vincristine therapy, 6 (11.11\%) patients relapsed during the first 6 months. Sustained remission at 6 months was seen in $15(27.78 \%)$ patients. Eleven (20.37\%), 9 (16.67\%), and $7(12.96 \%)$ patients had sustained remission at 12, 24, and 60 months, respectively (Figure 2). The number of patients having MCNS with mesangial proliferation (32/54) who achieved remission was significantly higher than that with FSGS $(22 / 54)(p=0.009)$.

The most frequently observed side-effects were abdominal distension and cramps, constipation, and change in the sense of taste. The occurrence of hair loss could be partly due to previous CYC therapy. None of these side-effects led to the discontinuation of treatment. The side-effects are indicated in Table 2. 


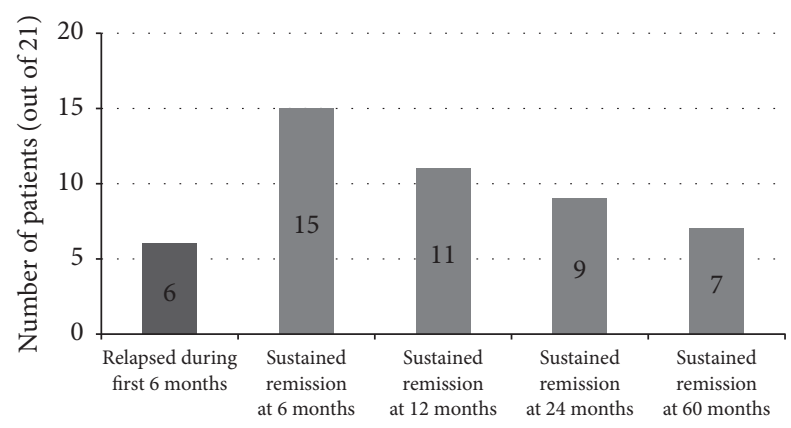

Figure 2: Patients who relapsed and remained in sustained remission for the first 60 months.

TABLE 2: Occurrence of side-effects.

\begin{tabular}{lc}
\hline Side-effect & Number of patients \\
\hline Vomiting & 7 \\
Weight loss & 4 \\
Diarrhoea & 6 \\
Bloating, abdominal pain, or cramps & 21 \\
Mouth ulcers & 3 \\
Headache & 4 \\
Hair loss & 38 \\
Constipation & 13 \\
Loss of appetite & 11 \\
Changes in sense of taste & 17 \\
Numbness and tingling in the hands and feet & 8 \\
Reversible bilateral ptosis & 3 \\
\hline
\end{tabular}

\section{Discussion}

In a review of randomized controlled trials on treatment strategies in SRNS, Hodson et al. conclude that calcineurin inhibitors such as cyclosporine increase the likelihood of complete or partial remission compared with placebo/no treatment or CYC [8]. Even though we used CYC as the firstline treatment for steroid resistance, treatment options for CYC resistance were limited due to the unavailability of CYA used in developed countries for such cases. This encouraged us to use vincristine as an alternative after CYC in the present series of patients.

Vincristine is a vinca alkaloid that has played an important role as a chemotherapeutic drug for malignant diseases. It exerts antitumor activity by preventing spindle microtubule formation to disable the aligning and moving of chromosomes. Composed of two multirings, vindoline and catherantine, it interacts with $\beta$-tubulin at a region adjacent to the GTP-binding site known as vinca domain $[9,10]$. Vincristine also is a potent inhibitor of Topoisomerase II [11].

The few previous studies done regarding vincristine and its effect on NS point towards the importance of vincristine use especially when the patient becomes resistant to steroids and second-line immunosuppressants. In 1994 Almeida et al. administered $1.5 \mathrm{mg} / \mathrm{m}^{2}$ of intravenous vincristine weekly for 8 weeks with simultaneous daily prednisolone for 4 weeks to children who were steroid resistant. With only 2 children out of 7 achieving complete remission, they concluded that their results do not encourage the use of vincristine [12]. However, Goonasekera et al. in 1998 highlighted the importance of reevaluating vincristine therapy as a potent alternative drug in patients with FSGS, based on their success with two children suffering from primary steroid-and cyclophosphamide-resistant FSGS who achieved complete remission with vincristine therapy [13]. A recent study by Kausman et al. where SDNS patients were treated with a longer regimen of vincristine reported significant reduction of relapse frequency and minimal side-effects. In addition, vincristine was also successful during subsequent relapses [14]. To add to these numbers, two children with SRNS and one child with SDNS out of 17 children achieved complete remission as reported by Krishnan et al. in 2006 [15].

The results of this study are more encouraging than the previously published literature in terms of the number of patients who achieved complete and partial remission. Most of the side-effects observed were transient and did not warrant discontinuation of the treatment. However, due to the use of steroids and CYC course prior to the vincristine therapy it is unclear if the achieved remission can be solely attributed to the action of vincristine. In spite of this, being inexpensive and reliable in patients who are noncompliant with oral medication and having fewer reversible side-effects can be stated as advantages of vincristine use [15].

How vincristine exerts its effects in nephrotic patients is still not elucidated. In an attempt to understand its action in adriamycin- (ADR-) induced nephropathy Yin et al. reported that vincristine can stabilize the actin cytoskeleton in the ADR-injured podocyte at a low dosage that does not disrupt microtubules. Their data also suggested that vincristine exerted this by suppressing overexpression of $\alpha 3 \beta 1$ integrin and focal adhesion kinase (FAK) [16]. This is important since inhibition of FAK activation or their deletion in podocytes has been found to protect against proteinuria and foot process effacement induced by glomerular injury [17]. Although this information provides an insight to vincristine's influence on nephropathy, further clarification of its therapeutic mechanism will definitely shed more light in this area. Thus it would be helpful to attract more attention to use it as a cheap and effective alternative treatment for NS.

\section{Conclusions}

In conclusion, it appears that vincristine is a potent and safe alternative treatment and should be considered in the treatment of SRNS. However a randomized controlled trial is required to ascertain whether it should be used in combination or as a single agent when treating SRNS.

\section{Disclosure}

The results of the initial 14 patients have been presented as an abstract at the International Pediatric Nephrology Association (IPNA) held in Adelaide, Australia, in 2004.

\section{Conflicts of Interest}

The authors declare that they have no conflicts of interest. 


\section{References}

[1] A. A. Eddy and J. M. Symons, "Nephrotic syndrome in childhood," The Lancet, vol. 362, no. 9384, pp. 629-639, 2003.

[2] A. Bagga, A. Gulati, S. Gulati, K. P. Mehta, and M. Vijayakumar, "Management of steroid resistant nephrotic syndrome," Indian Pediatrics, vol. 46, no. 1, pp. 35-47, 2009.

[3] A. Zagury, A. L. Oliveira, J. A. Montalvão et al., "Steroidresistant idiopathic nephrotic syndrome in children: long-term follow-up and risk factors for end-stage renal disease," Jornal Brasileiro de Nefrologia, vol. 35, no. 3, pp. 191-199, 2013.

[4] Symposium on Pediatric Nephrology, "Primary nephrotic syndrome in children: clinical significance of histopathologic variants of minimal change and of diffuse mesangial hypercellularity," Kidney International, vol. 20, no. 6, pp. 765-771, 1981.

[5] A. A. Abeyagunawardena, "Childhood nephrotic syndrome: steroid sensitive, steroid resistant and beyond," Sri Lanka Journal of Child Health, vol. 38, no. 4, 2009.

[6] D. Habashy, E. M. Hodson, and J. C. Craig, "Interventions for steroid-resistant nephrotic syndrome: a systematic review," Pediatric Nephrology, vol. 18, no. 9, pp. 906-912, 2003.

[7] C. Plank, V. Kalb, B. Hinkes, F. Hildebrandt, O. Gefeller, and W. Rascher, "Cyclosporin a is superior to cyclophosphamide in children with steroid-resistant nephrotic syndrome-A randomized controlled multicentre trial by the Arbeitsgemeinschaft für Pädiatrische Nephrologie," Pediatric Nephrology, vol. 23, no. 9, pp. 1483-1493, 2008.

[8] E. Hodson, S. Wong, N. Willis, and J. Craig, Interventions for Idiopathic Steroid-Resistant Nephrotic Syndrome in Children, 2016.

[9] M. A. Jordan and L. Wilson, "Microtubules as a target for anticancer drugs," Nature Reviews Cancer, vol. 4, no. 4, pp. 253265, 2004.

[10] S. S. Rai and J. Wolff, "Localization of the vinblastine-binding site on $\beta$-tubulin," Journal of Biological Chemistry, vol. 271, no. 25, pp. 14707-14711, 1996.

[11] A. Skladanowski, M.-G. Côme, M. Sabisz, A. E. Escargueil, and A. K. Larsen, "Down-regulation of DNA topoisomerase II $\alpha$ leads to prolonged cell cycle transit in G2 and early $\mathrm{M}$ phases and increased survival to microtubule-interacting agents," Molecular Pharmacology, vol. 68, no. 3, pp. 625-634, 2005.

[12] M. P. Almeida, H. A. Almeida, and F. Coelho Rosa, "Vincristine in steroid-resistant nephrotic syndrome," Pediatric Nephrology, vol. 8, no. 1, pp. 79-80, 1994.

[13] C. D. A. Goonasekera, A. B. Koziell, S. A. Hulton, and M. J. Dillon, "Vincristine and focal segmental sclerosis: do we need a multicentre trial?" Pediatric Nephrology, vol. 12, no. 4, pp. 284289, 1998.

[14] J. Y. Kausman, L. Yin, C. L. Jones, L. Johnstone, and H. R. Powell, "Vincristine treatment in steroid-dependent nephrotic syndrome," Pediatric Nephrology, vol. 20, no. 10, pp. 1416-1419, 2005.

[15] R. G. Krishnan, M. G. Coulthard, and N. E. Moghal, "Is there a role for vincristine in nephrotic syndrome?" Pediatric Nephrology, vol. 21, no. 4, p. 597, 2006.

[16] L. Yin, Y. Mao, H. Song, Y. Wang, W. Zhou, and Z. Zhang, "Vincristine alleviates adriamycin-induced nephropathy through stabilizing actin cytoskeleton," Cell \& Bioscience, vol. 7, no. 1, 2017.
[17] H. Ma, A. Togawa, K. Soda et al., "Inhibition of podocyte FAK protects against proteinuria and foot process effacement," Journal of the American Society of Nephrology, vol. 21, no. 7, pp. 1145-1156, 2010. 


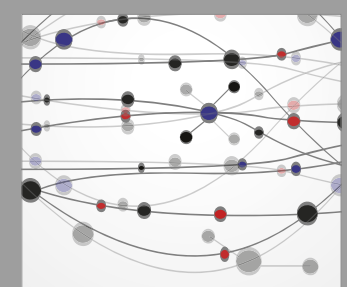

The Scientific World Journal
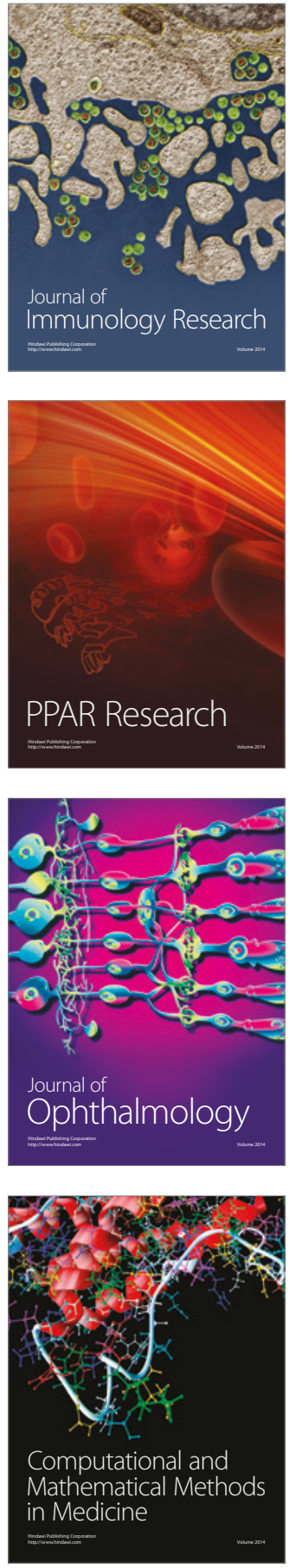

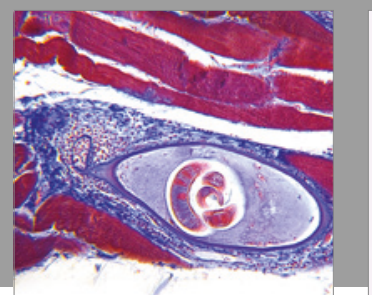

Gastroenterology Research and Practice
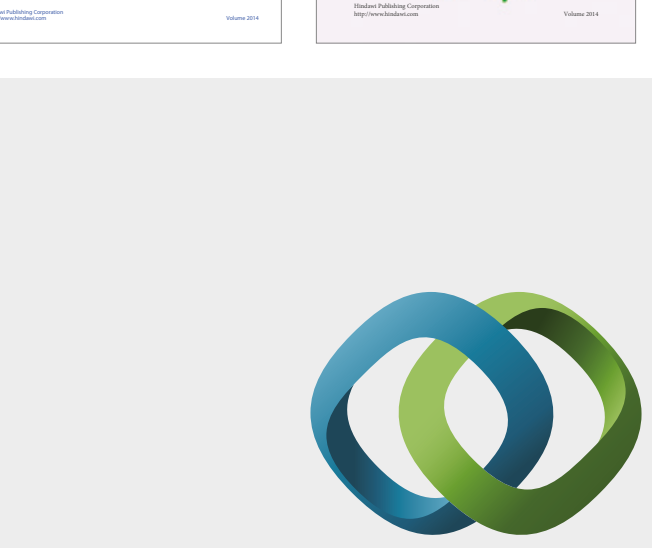

\section{Hindawi}

Submit your manuscripts at

https://www.hindawi.com
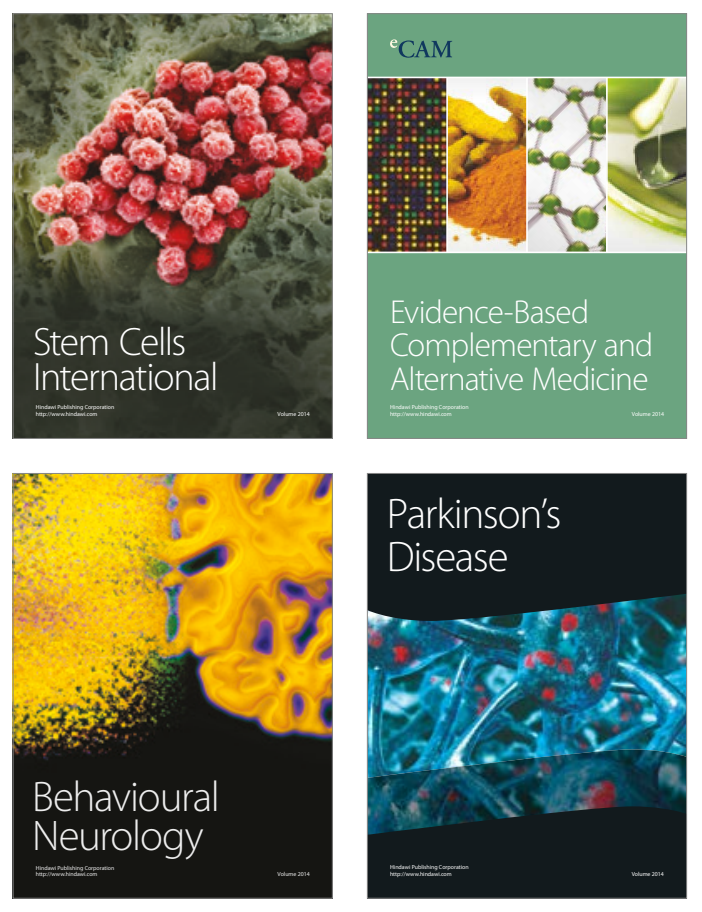
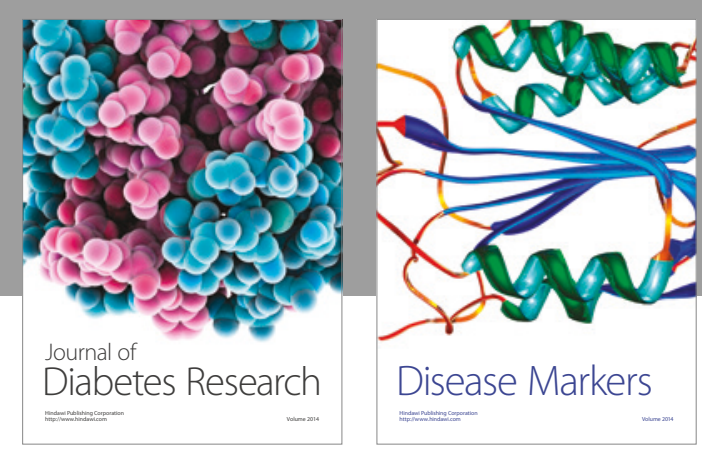

Disease Markers
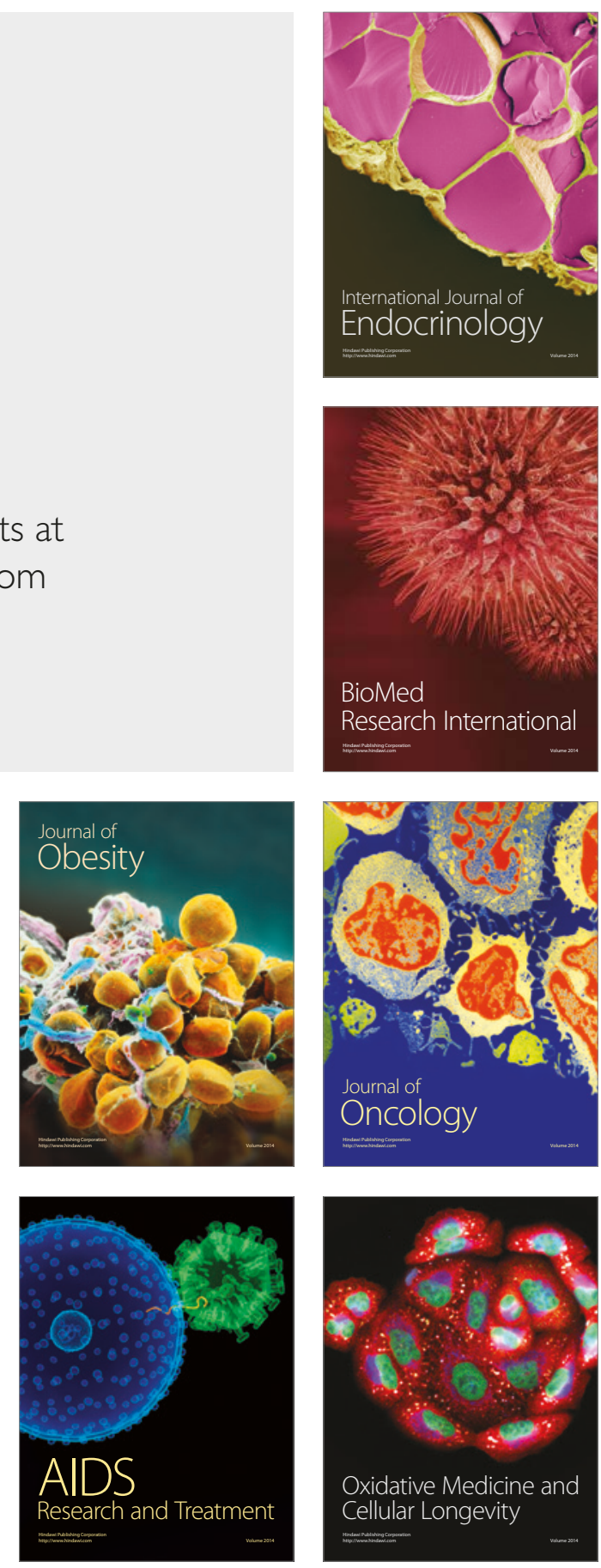